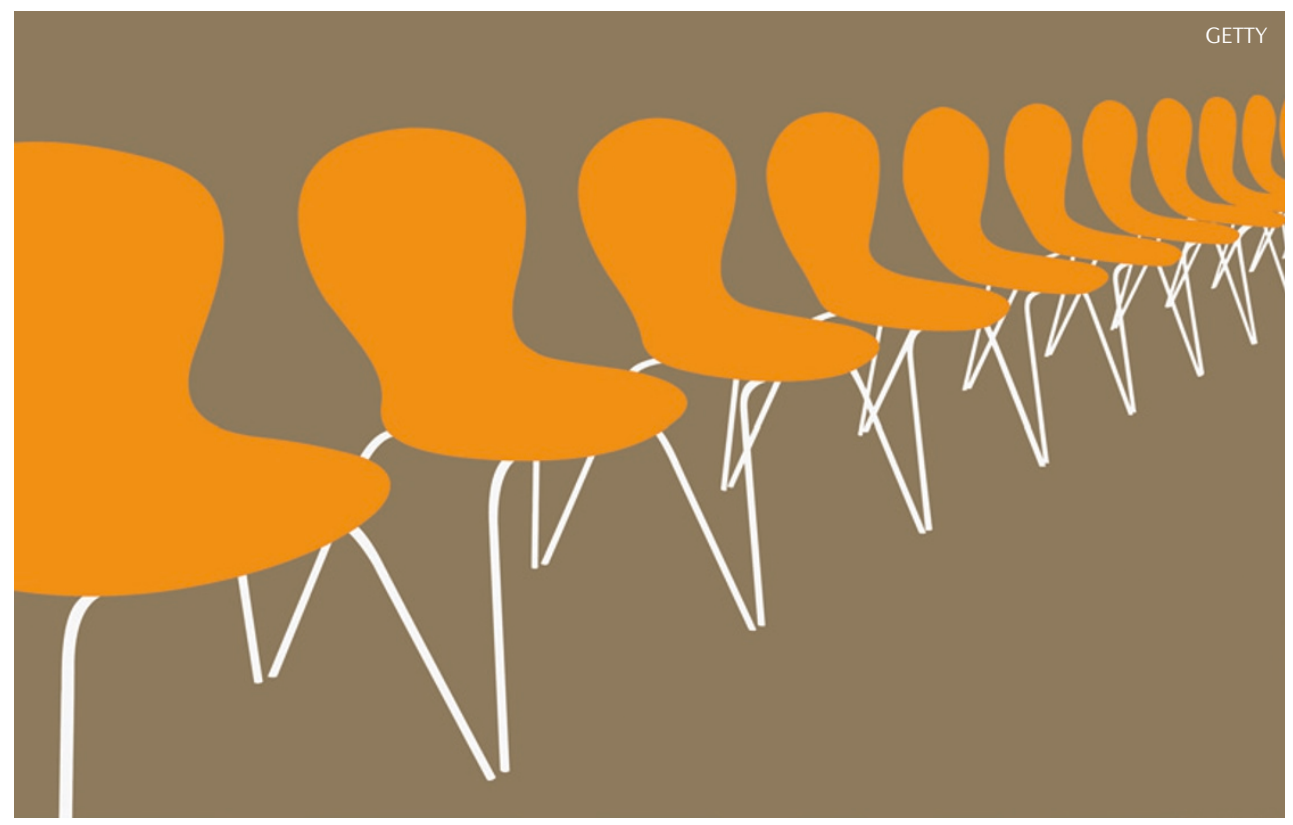

$\Rightarrow$ DEVELOPMENT

\title{
Musical chairs at the epithelium
}

The development and homeostasis of epithelial tissues is tightly controlled through the regulation of cell growth, proliferation and cell death. For example, the induction of apoptosis, followed by the extrusion of apoptotic cells, was previously shown to prevent overcrowding in growing epithelial membranes. Now, two studies describe a new control mechanism in epithelial homeostasis that involves delamination of live cells in response to overcrowding.

Eisenhoffer et al. observed the extrusion of non-apoptotic cells in human colon tissue, developing zebrafish epidermis and an overgrown epithelial monolayer of Madin-Darby canine kidney (MDCK) cells. Interestingly, the number of delaminating live cells was increased at surfaces with high cell densities. Moreover, experimental induction of overcrowding of an MDCK cell monolayer indicated that overcrowding correlates with increased delamination of live cells. This process was found to involve the activation of the small GTPase RHO downstream of sphingosine-1-phosphate (S1P) signalling, leading to actin ring contraction, which squeezed the cells out of the epithelium. In addition, overcrowding was found to signal the induction of live-cell delamination through stretch-activated ion channels. In zebrafish epidermis, the ion channel Piezo 1 is essential for the homeostatic extrusion of non-apoptotic cells, as deletion of Piezo 1 led to epidermal overgrowth, thus highlighting a potential link between live-cell delamination and tumorigenesis.
Marinari et al. observed the delamination of live cells from crowded regions of an epithelium during the development of the dorsal thorax in the pupae of Drosophila melanogaster. By combining imaging studies with computational simulations, they concluded that live-cell delamination results from stochastic cell rearrangements in a crowded epithelium, and that it acts like a homeostat to optimize cell packing. Indeed, genetically induced cell growth increased cell delamination, whereas delamination was completely inhibited in tissues with defective growth. Moreover, live-cell delamination was shown to be independent of cell division, was not affected by the expression of an inhibitor of apoptosis and was visibly distinct from the extrusion of dying cells from the tissue. Finally, cells that had lost their apical area and 3-4 cell-cell junctions were pushed out of the tissue through a force excerted by a contractile myosin II purse-string, which formed among neighbouring cells.

Together, these studies reveal a new mechanism that maintains the integrity of epithelial tissues during development and homeostasis. Further research will show whether dysregulation of live-cell delamination is involved in the development of diseases such as cancer.

Maria Papatriantafyllou

ORIGINAL RESEARCH PAPERS Eisenhoffer, G. T. et al. Crowding induces live cell extrusion to maintain homeostatic cell numbers in epithelia. Nature 15 Apr 2012 (doi:10.1038/nature10999)|

Marinari, E. et al. Live-cell delamination counterbalances epithelial growth to limit tissue overcrowding. Nature 15 Apr 2012 (doi:10.1038/nature10984) 